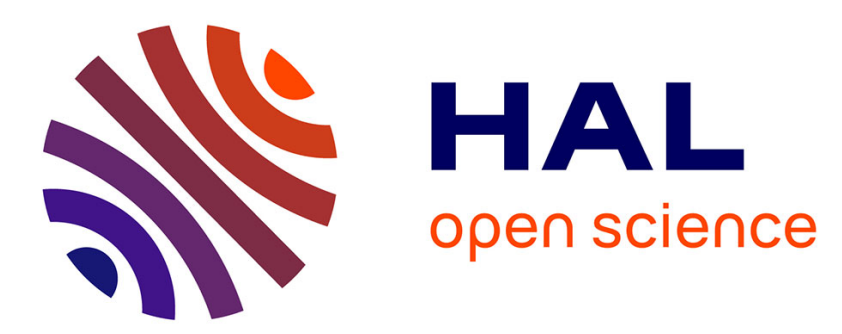

\title{
Evaluation of Invisible Physical and Mental Exertion from CT Scan Operation in Saudi Arabian Hospitals
}

\author{
Saad Aldoihi, Omar Hammami
}

\section{To cite this version:}

Saad Aldoihi, Omar Hammami. Evaluation of Invisible Physical and Mental Exertion from CT Scan Operation in Saudi Arabian Hospitals. the International Conference on Control, Decision and Information Technologies, Apr 2019, Paris, France. hal-02191122

\section{HAL Id: hal-02191122 \\ https://hal.science/hal-02191122}

Submitted on 23 Jul 2019

HAL is a multi-disciplinary open access archive for the deposit and dissemination of scientific research documents, whether they are published or not. The documents may come from teaching and research institutions in France or abroad, or from public or private research centers.
L'archive ouverte pluridisciplinaire HAL, est destinée au dépôt et à la diffusion de documents scientifiques de niveau recherche, publiés ou non, émanant des établissements d'enseignement et de recherche français ou étrangers, des laboratoires publics ou privés. 


\section{Evaluation of Invisible Physical and Mental Exertion from CT Scan Operation in Saudi Arabian Hospitals}

\author{
Saad Aldoihi \\ Computer Science and Systems Engineering \\ ENSTA PARISTECH \\ Palaiseau 91762 Cedex, France \\ saad.aldoihi@ensta-paristech.fr
}

\author{
Omar Hammami \\ Computer Science and Systems Engineering \\ ENSTA PARISTECH \\ Palaiseau 91762 Cedex, France \\ Hammami@ensta-paristech.fr
}

\begin{abstract}
Advancement in science and technology has increased work exertion tremendously. Many researchers and professionals have noted the importance of work exertion in correlation with the work environment. However, there is no mention of the principle of invisible exertion correlated with the current advancement of science and technology. This paper evaluates the invisible physical and mental exertion for CT scan technicians. Fifty-seven CT scan technicians were surveyed to determine the extent of their invisible physical and mental exertion. The overwhelming majority of technicians reported significant exertion while operating the CT scan. The invisible physical and mental exertion occurred differently in accordance with demographic characteristics. Gender is a significant factor, where male technicians reported more physical invisible exertion than female technicians. The invisible physical exertion occurred more than invisible mental exertion.
\end{abstract}

Index Terms-invisible exertion, CT scan invisible exertion, CT scan overload effects

\section{INTRODUCTION}

Heuristics is a popular method to evaluate system and product usability, and it proposes improvements during and after the development. A key component of the success and rising popularity of heuristics is its low cost and effectiveness.

In academia, there are 152 usability attributes [1]. As a customary practice in industry and academia, usability is constructed from a list of attributes to constitute the dimensions of the cohesive whole. Geisen and Bergstrom [2] referred to these dimensions as metrics for evaluation. It is clear from the literature that usability lacks uniformity and unity of dimensions, which has caused some ambiguity among new researchers.

However, it is vitally important to state why usability diverges in dimensions. An important reason is that usability is used to evaluate various products and systems that hold different execution goals and purposes. According to Ferre et al. [3], usability can only be defined in accordance with the intended system and the intended users. They illustrated their argument regarding a museum kiosk where the dimensions must emphasize minimum training since kiosk users are most likely to use it only once in their lifetime.
Similarly, usability attributes should be representative of the overall environment where the larger scope must be counted as an attribute. For instance, the incorporated usability considers the local environment of the work, including the managerial target and departmental duty that measures the invisible exertion, which can contribute to and affect usability. The obvious objective is to use the usability evaluation to consider human factors that are associated with the work and managerial environments. When evaluating usability attributes, there is a clear indication that they lack an exertion evaluation, such as for physical and mental exertion. Consequently, many authors [4] [5] [6] have included NASA-LTX for a usability evaluation as a substitute for physical and mental exertion, which is lacking in popular heuristic methods.

One of the ISO 9241 usability evaluation settings is "context of use." In essence, it implies that evaluation usability must consider the work atmosphere in which the device or system would operate. For instance, when evaluating a newly developed CT scan, the natural everyday busy setting of an over-crowded and understaffed hospital must be considered, including the management target and duty expected from the operators. In other words, exertion delivered from any source should be included in an operator's human factor and usability evaluations. Dimensional attributes such as safety, operator satisfaction, and productivity are the essence that constitutes the overall usability [7]. Furthermore, impeding attributes that consider the organizational context would add more accuracy and validity of the overall usability.

The overall objective of this study is to identify invisible exertion existence while operating CT and to identify whether the Radiology Department duty and management targets can be transformed into invisible physical and mental exertion. To the best of our knowledge, there is no existing study that measures the invisible exertion of CT scan technicians and correlates the management process to influence technician exertion. The area of this research has not been exploited by other researchers; thus, the authors hope that this study will open a new area of research that considers invisible exertion of whatever kind and correlates it to the overall evaluation. 


\section{BACKGROUND}

\section{A. Usability as a factor in medical devices}

From the beginning, medical devices have been implemented on the premise of assisting the examiner by providing vital information that could alter the examiner's decisions regarding the patients. As the devices gain more computing power and advanced sensing technology, more functionalities and connectivity with other devices present more challenges to the novice user. Consequently, evaluation methods have been suggested from different fields. In recent years, usability evaluations have dramatically shifted focus to medical devices.

Designing usability for medical devices is not like other electronic devices for the following reasons. Medical devices provide data on decisions regarding the actions of doctors. Medical device usability has a limit, where user characteristics and preferences count for the overall outcome. Aldoihi and Hammami [6] showed that CT scan operators indeed differ in perceiving the usability attributes according to gender and years of experience.

\section{B. Cognitive and Physical Exertion}

Cognitive load is deeply involved in psychology. An obvious sign of such involvement is that cognitive load is only clarified through psychology or behavioral lenses [8]. Key components of cognitive load are the nature of the work and how memory can correspond with it [9]. Mental load has been perceived by early psychological theorists as a multi-dimensional phenomenon where the interpretation of phenomena comes as a result of the outcomes of the interaction between subjective individual characteristics and objective task characteristics [10] [11]. Subsequently, mental load resulting from indirect (invisible) work has not been properly studied.

Mental load studies have heavily concentrated on the direct aspects and have somehow neglected indirect aspects. It is essential to include invisible elements to understand the overall characteristics of the mental load since the mental load is multi-dimensional by nature. Mental load exists whenever there are intractable tasks that demand a great deal of control. There are three main aspects of invisible physical and mental loads, which are task complexity, management requirements, and pressure of resources (see Fig. 1).

Another extremely important load is the physical load. For CT scan technicians, there is a considerable amount of physical load during the operation of the machine. Nonetheless, there are many tasks performed by the technicians where the physical load is unavoidable. Such tasks are the framework of this study. For instance, it is a customary practice in Saudi Arabia that CT technicians help the bedridden patient transfer to the CT table. Literature has demonstrated the effect of overload on radiologists on many fronts. Various studies have explored the risk factors associated with excessive loads [12] [13] [14]. Bruni et al. [15] noted that considerable discrepancies in interpretation are demonstrated in the late shift for radiologists as opposed to the starting shift. It is critically important to explore and identify the effect of the invisible load on

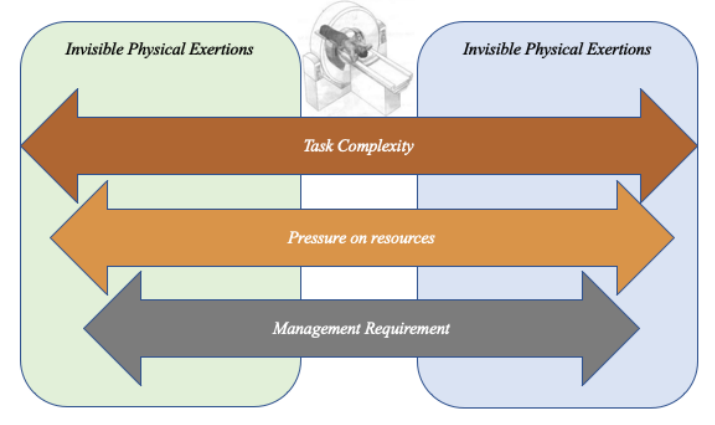

Fig. 1: A conceptual framework of the physical and mental exertion sources

radiologists so that researchers and practitioners can propose reduction procedures.

\section{METHOD}

For this research, the authors developed a survey questionnaire that measures invisible exertion while operating the CT. Such exertions can be unintended and come from the inner work environment.

1) Participants: The population of the study comprises CT scan technicians who operate the machine on a daily basis and who are geographically located in Saudi Arabia. One vital variable dimension that must be distinguished is the work sector. In Saudi Arabia, the healthcare sector is divided into two categories. That is a public and private sector. It is essential to distinguish these two groups within the demographic characteristics. In total, 57 technicians participated in this study. The participants are all CT scan technicians who, at the time of the study, were working in Saudi Arabia in either the public or private sector. Participants were invited to the study by either online questionnaire link or by a telephone call. See Table 1 for more details of the demographic characteristics.

TABLE I: Participant Demographic Characteristics

\begin{tabular}{|l|l|r|}
\hline Variable & & Total Percentage \\
\hline \multirow{4}{*}{ Gender } & Male & $50.90 \%$ \\
\cline { 2 - 3 } Total & Female & $49.10 \%$ \\
\hline \multirow{5}{*}{ Age } & & $100 \%$ \\
\hline Total & $20-29$ & $77.20 \%$ \\
\cline { 2 - 3 } & $30-39$ & $15.80 \%$ \\
\cline { 2 - 3 } & $40-49$ & $3.50 \%$ \\
\cline { 2 - 3 } & $50+$ & $3.50 \%$ \\
\hline \multirow{4}{*}{ Educational level } & & $100 \%$ \\
\hline Total & Diploma & $3.50 \%$ \\
\hline \multirow{4}{*}{ Years of Radiology Experience } & Bachelor & $86.00 \%$ \\
\cline { 2 - 3 } & Master & $10.50 \%$ \\
\hline Total & & $100 \%$ \\
\hline \multirow{3}{*}{ WorkingSector } & $0-3$ years & $68.40 \%$ \\
\cline { 2 - 3 } & $4-7$ years & $8.80 \%$ \\
\hline Total & $12+$ & $10.50 \%$ \\
\hline \multirow{2}{*}{} & & $12.30 \%$ \\
\cline { 2 - 3 } & Public Sector & $87.70 \%$ \\
\cline { 2 - 3 } & & $100 \%$ \\
\hline
\end{tabular}


2) Survey method: After evaluating the causes of invisible physical and mental exertion, a field visit was conducted to King Saudi Medical City (KSMC) to gather and observe technician exertion. Due to the excessive particularity of the measured variables, a questionnaire was developed to suit the special particularity. The questionnaire was sent to technicians across Saudi Arabia, asking them to respond to six statements regarding their views on physical and mental activities while operating a CT. Three statements represent physical exertion (PRQ), and the other three represent mental exertion (MRQ). The statements were as follows:

1) PRQ1: I have helped to transfer a bedridden patient from the hospital bed to the radiology table,

2) PRQ2: It is my duty to prepare the examination room for receiving the next patient,

3) PRQ3: It is my duty to prepare for contrast media administration as needed,

4) MRQ1: I think that I work in an understaffed environment,

5) MRQ2: I think that the department needs more CT scan machines,

6) MRQ3: I think that management targets are unreasonable (not in alignment with existing resources).

In addition, open-ended questions were added after each statement as optional commentary feedback. A self-rating questionnaire was created based on a five-point Likert scale.

\section{RESULTS}

\section{A. Invisible Physical Exertions}

Invisible physical exertion is presented clearly in Fig. 2. The research statements demonstrate the invisible physical exertion through the activities that are required to operate a CT from three dimensions, which are PRQ1-PRQ3, as stated above. For transferring the bedridden patient to the CT table, 29 (50.9\%) technicians responded with "always." "Usually" constituted $17(29.8 \%)$ responses. "Sometimes" constituted $10(17.5 \%)$ responses, whereas "never" constituted only 1 $(1.8 \%)$ response. For preparing the examination room for the next patient, $43(75.4 \%)$ responded with "always." "Usually" constituted $10(17.5 \%)$ responses. "Sometimes" constituted 4 (7\%) responses. For preparing and administrating the contrast media, 38 (66.7\%) responded with "always." "Usually" constituted $13(22.8 \%)$ responses. "Sometimes" constituted 4 (7\%) responses. "Rarely" and "never" constituted 1 (1.8\%) response.

\section{B. Invisible Mental Exertions}

Invisible mental exertion is presented clearly in Fig. 3. The research questions demonstrate the invisible mental exertion through activities required to operate a CT from three dimensions, which are MRQ1-MRQ3, as stated above. For working in an understaffed environment, 14 (24.6\%) technicians responded with "strongly agree." "Agree" constituted $19(33.3 \%)$ responses. "Neutral" constituted 20 (35.1\%) responses. "Disagree" constituted 4 (7\%) responses. For suitability of the number of CT machines, $22(38.6 \%)$ techni-

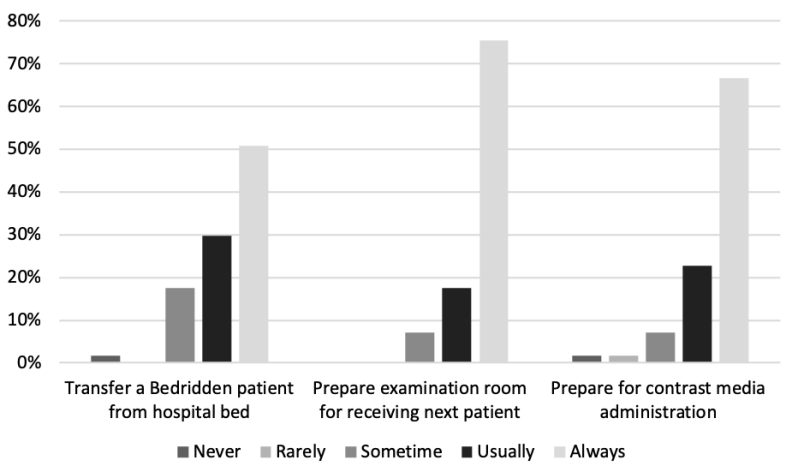

Fig. 2: Physical exertion responses

cians responded with "strongly agree." "Agree" constituted 18 (31.6\%) responses. "Neutral" constituted 7 (12.3\%) responses. "Disagree" constituted 8 (14\%) responses. "Strongly disagree" constituted $2(3.5 \%)$ responses. For management alignment with existing resources, $14(24.6 \%)$ technicians responded with "strongly agree." "Agree" constituted 19 (33.3\%) responses. "Neutral" constituted 14 (24.6\%) responses. "Disagree" constituted $10(17.5 \%)$ responses.

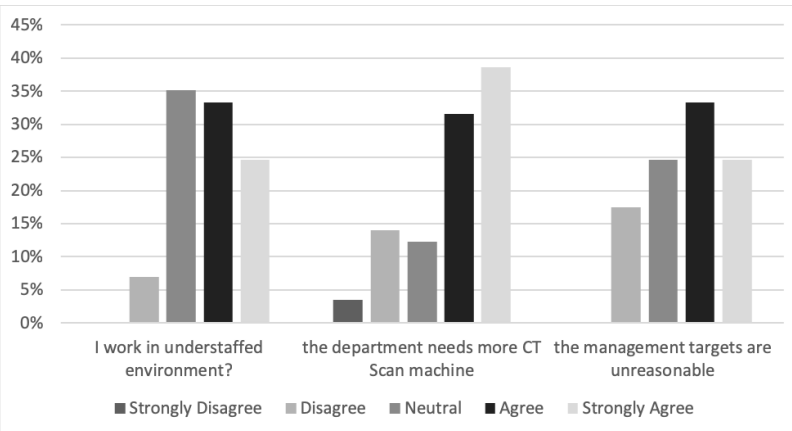

Fig. 3: Mental exertion responses

\section{Demographic Characteristics: Physical and Mental}

1) Gender: Figure 4 shows the proportion of difference between male and female respondents. For the physical exertion statement in PRQ1, $62.1 \%$ of male technicians indicated that they always contribute physically by transferring the bedridden patient to the CT table, in contrast to only $39.3 \%$ of female technicians. Interestingly, $3.6 \%$ of female technicians stated that they never helped or contributed to transferring the bedridden patients to the CT table. In response to PRQ2, $89.7 \%$ of male technicians indicated that they have always prepared the exam room for the next patient, as opposed to $60.7 \%$ of female technicians. In response to PRQ3, 79.3\% of male technicians indicated that they always prepared the contrast media for the next patient, in contrast to $53.6 \%$ of female technicians. Interestingly, $7.2 \%$ of female technicians responded with "never" or "rarely."

In response to mental exertion statements, in MRQ1, 34.5\% of male technicians indicated that they work in an understaffed 
environment, as opposed to only $14.3 \%$ of female technicians. In response to MRQ2, 48.3\% of male technicians indicated that the department needs more CT scanners to accommodate the patient overload, compared to $28.6 \%$ of female technicians. In response to MRQ3, 27.6\% of male technicians indicated that management targets are not aligned with existing resources, compared with $21.4 \%$ of female technicians.

2) Age: Age is a fundamental factor in determining physical and mental exertion. Figure 5 shows the proportion of difference between age groups from 20 to 29,30 to 39 , 40 to 49 , and over 50 . In response to the physical exertion statement in PRQ1, 43.2\% of the 20 to 29 age group indicated they always contribute physically to transferring the bedridden patients to the CT table. Similarly, $66.7 \%$ of the 30 to 39 age group indicated that they always contribute physically, whereas all of the 40 to 49 and over 50 age groups indicated that they always contribute physically. In response to PRQ2, 75\% of the 20 to 29 age group responded with "always" for preparing the examination room for the next patient, compared to $66.7 \%$ of the 30 to 39 age group. Both the 40 to 49 and over 50 age groups stated that they always prepare the examination room for the next patient. In response to PRQ3, 63.6\% of the 20 to 29 age group reported that they prepare and administrate the media contrast for the next patient, compared to $77.8 \%$ of the 30 to 39 age group, $50 \%$ of the 40 to 49 age group, and $100 \%$ of the over 50 age group.

In response to the statements regarding mental exertion, in MRQ1, $20.5 \%$ of the 20 to 29 age group strongly agree that they work in an understaffed environment in comparison with $33.3 \%$ of the 30 to 39 age group, $50 \%$ of the 40 to 49 age group, and $50 \%$ of the over 50 age group. In response to MRQ2, $38.6 \%$ of the 20 to 29 age group reported that they strongly agree that they work in the Radiology Department with fewer CT scans in operation, in contrast to $33.3 \%$ of the 30 to 39 age group and $50 \%$ of both the 40 to 49 and over 50 age groups. In response to MRQ3, 20.5\% of the 20 to 29 age group reported that the management targets are not aligned with the existing resources, in comparison to $33.3 \%$ of the 30 to 39 age group and $50 \%$ of both the 40 to 49 and over 50 age groups.

3) Years of radiology experience: Years of experience is a dominant factor in determining the invisible physical and mental exertion. Figure 6 shows the proportion of difference between 0 to 3 years, 4 to 7 years, 8 to 11 years, and more than 12 years. In response to the physical exertion statement in PRQ1, $46.2 \%$ of those with 0 to 3 years of experience reported that they always contribute physically to transferring bedridden patients to the CT table, in comparison to $20 \%$ of those with 4 to 7 years of experience, $66.7 \%$ of those with 8 to 11 years of experience, and $85.7 \%$ of those with more than 12 years of experience. In response to PRQ2, 74.4\% of those with 0 to 3 years of experience reported that they always prepare the examination room for the next patient, in contrast to $80 \%$ of those with 4 to 7 years of experience, $66.7 \%$ of those with 8 to 11 years of experience, and $85.7 \%$ of those with more than 12 years of experience. In response to PRQ3,
$61.5 \%$ of those with 0 to 3 years of experience reported that they prepare and administrate the media contrast for the next patient, in comparison to $80 \%$ of those with 4 to 7 years of experience, $66.7 \%$ of those 8 to 11 years of experience, and $85.7 \%$ of those with more than 12 years of experience.

In response to the mental exertion statement in MRQ1, $17.9 \%$ of those with 0 to 3 years of experience reported that they strongly agreed that they work in an understaffed environment in contrast to $40 \%$ of those with 4 to 7 years of experience, $33.3 \%$ of those with 8 to 11 years of experience, and $42.9 \%$ of those with more than 12 years of experience. In response to MRQ2, 41\% of those with 0 to 3 years of experience reported that they strongly agree that they work in a Radiology Department with fewer CT scans in operation, compared to $20 \%$ of those with 4 to 7 years of experience, $50 \%$ of those with 8 to 11 years of experience, and $28.6 \%$ of those with more than 12 years of experience. In response to MRQ3, $23.1 \%$ of those with 0 to 3 years of experience reported that the management targets are not aligned with existing resources, in contrast to $33.3 \%$ of those with 8 to 11 years of experience and $42.9 \%$ of those with more than 12 years of experience.

\section{CONCLUSION}

Generally, the findings suggest that there is indeed invisible physical and mental exertion associated with the operation of CT scans. Regarding the agreement rate in response to the physical statement in PRQ1, 80.7\% of radiologists confirmed that they commonly transfer bedridden patients to the CT table. Therefore, manufacturers should pay extra attention when designing the machine because meeting the technical requirements is not enough for delivering a suitable machine that meets all work environment requirements. It is fundamentally ideal if the manufacturing standards cover customization that meets the lowest end-user specifications. Fundamentally, patients are the core reason for the existence of the machine. Therefore, empowerment of the patient's current condition during the exam is highly sought after by many patients, especially during the movement of bedridden patients from and to the CT table. The current CT scan machine on the market lacks the basic habilitation capability that is needed to empower those who are bedridden. The agreement rate in response to the physical statement in PRQ2 indicates that $93 \%$ of radiologists confirmed that they frequently prepare the examination room for each patient. Moreover, the agreement rate in response to the physical exertion statement in PRQ3, 89.5\% of the radiologists confirmed that they frequently prepare and administrate the contrast media to patients. The findings imply that invisible physical exertion occurs more than invisible mental exertion. The agreement is $57.9 \%$ for the mental exertion statement in MRQ1, 70.2\% for MRQ2, and 57.9\% for MRQ3. Two-thirds of radiologists agree with the mental exertion statement. Nonetheless, they almost consensually agree with the invisible physical exertion general statements. As future work, the authors intend to propose a human-centered system engineering model to integrate usability and exertions at the very beginning of the design flow. 


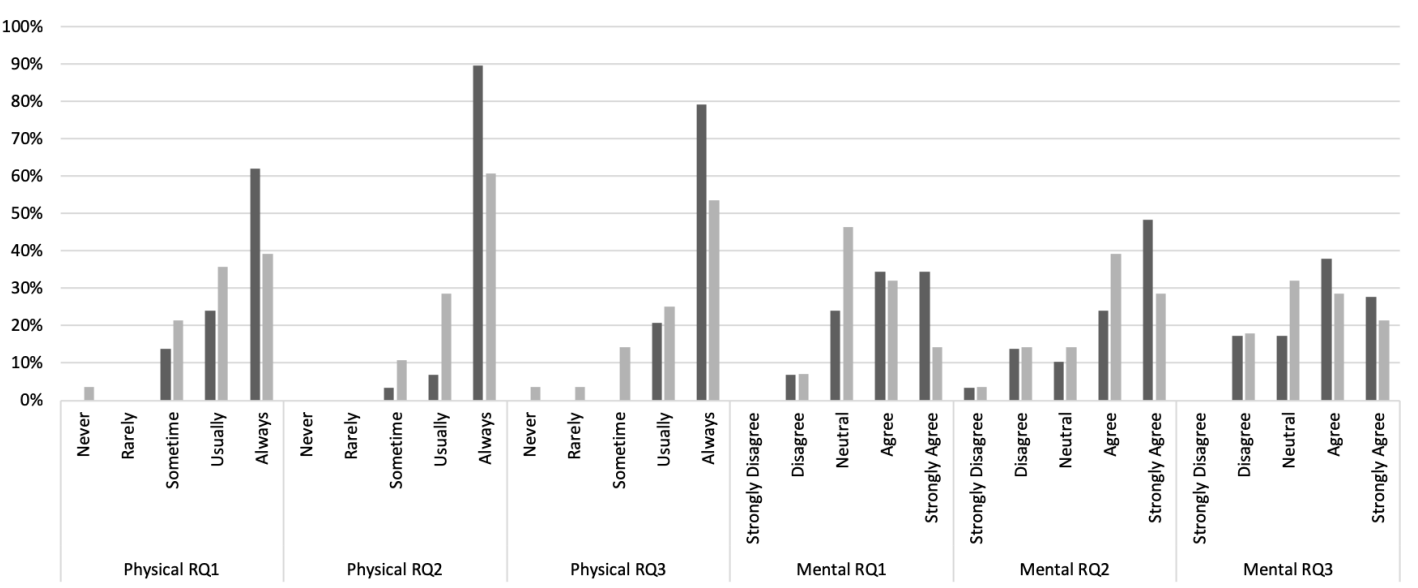

Fig. 4: Physical and mental exertion respond based on gender

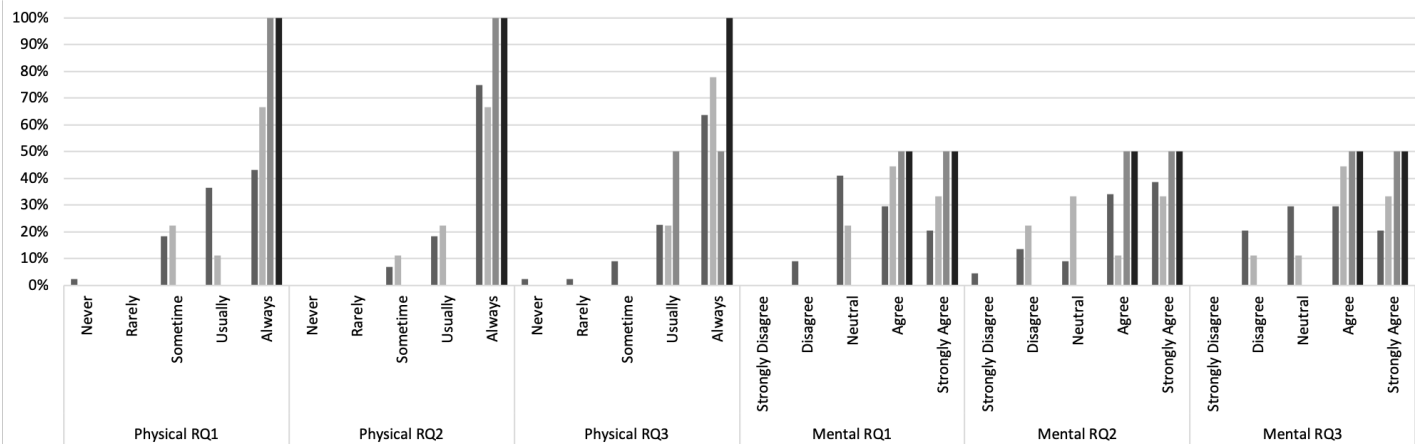

Fig. 5: Physical and mental exertion response based on age

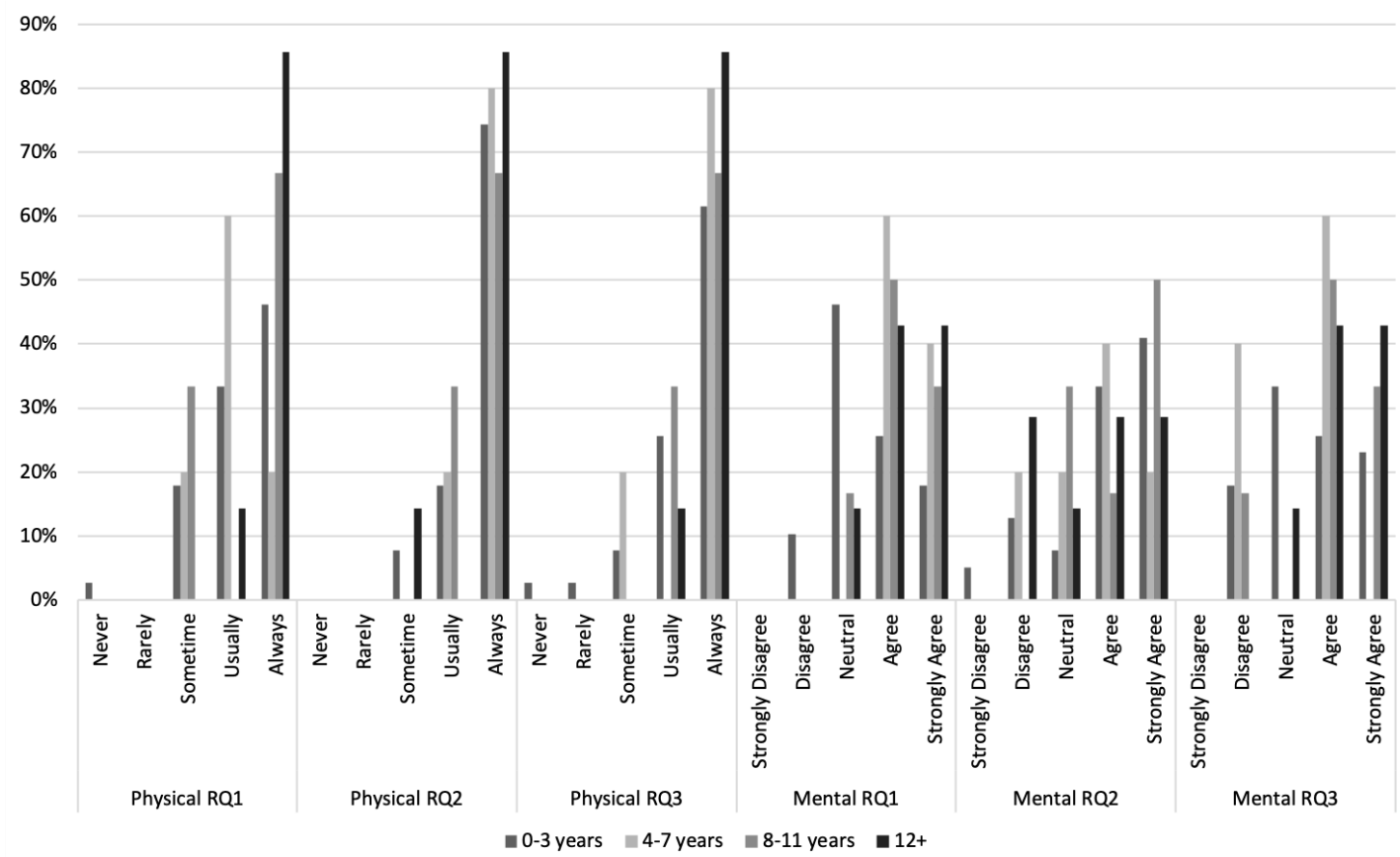

Fig. 6: Physical and mental exertion response based on years of experience 


\section{ACKNOWLEDGMENTS}

The authors thank the Saudi Health Ministry for providing access to the hospitals and employees. A special thanks goes to all participants who made the time and effort to participate in this study.

\section{REFERENCES}

[1] Dubey, S. K., and Rana, A. (2010). Analytical roadmap to usability definitions and decompositions. International Journal of Engineering Science and Technology, 2(9), 4723-4729.

[2] Geisen, E., and Bergstrom, J. R. (2017). Usability testing for survey research. Morgan Kaufmann.

[3] Ferré, X., Juristo, N., Windl, H., and Constantine, L. (2001). Usability basics for software developers. IEEE software, 18(1), 22-29.

[4] Longo, L., and Kane, B. (2011, June). A novel methodology for evaluating user interfaces in health care. In Computer-Based Medical Systems (CBMS), 2011 24th International Symposium on (pp. 1-6). IEEE.

[5] Ramkumar, A., Song, Y., Niessen, W. J., and Stappers, P. J. (2016, June). Design Issues of the Existing Radiotherapy Segmentation Software. In Proceedings of the International Symposium on Human Factors and Ergonomics in Health Care (Vol. 5, No. 1, pp. 1-8). Sage India: New Delhi, India: SAGE Publications.

[6] Aldoihi, S., and Hammami, O. (2018, July). Evaluation of CT Scan Usability for Saudi Arabian Users. In 2018 International Conference on Computer, Information and Telecommunication Systems (CITS) (pp. 1-5). IEEE.

[7] Helander, M. (2005). A guide to human factors and ergonomics. Crc Press.

[8] Moreno, R. E., and Park, B. (2010). Cognitive load theory: Historical development and relation to other theories.

[9] Sweller, J., Ayres, P., and Kalyuga, S. (2011). Measuring cognitive load. In Cognitive load theory (pp. 71-85). Springer, New York, NY.

[10] Campbell, D. J. (1988). Task complexity: A review and analysis. Academy of management review, 13(1), 40-52.

[11] Wood, R. E. (1986). Task complexity: Definition of the construct. Organizational behavior and human decision processes, 37(1), 60-82.

[12] Hanna, T. N., Zygmont, M. E., Peterson, R., Theriot, D., Shekhani, H., Johnson, J. O., and Krupinski, E. A. (2018). The Effects of Fatigue From Overnight Shifts on Radiology Search Patterns and Diagnostic Performance. Journal of the American College of Radiology.

[13] Krupinski, E. A., Berbaum, K. S., Caldwell, R. T., Schartz, K. M., Madsen, M. T., and Kramer, D. J. (2012). Do long radiology workdays affect nodule detection in dynamic CT interpretation?. Journal of the American College of Radiology, 9(3), 191-198.

[14] Krupinski, E. A., Berbaum, K. S., Caldwell, R. T., Schartz, K. M., and Kim, J. (2010). Long radiology workdays reduce detection and accommodation accuracy. Journal of the American College of Radiology, 7(9), 698-704.

[15] Bruni, S. G., Bartlett, E., and Yu, E. (2012). Factors involved in discrepant preliminary radiology resident interpretations of neuroradiological imaging studies: a retrospective analysis. American Journal of Roentgenology, 198(6), 1367-1374. 